\title{
Comparative proteomic profiling identifies potential prognostic factors for human clear cell renal cell carcinoma
}

\author{
XIANG SUN ${ }^{1 *}$, HONGWEI ZHANG ${ }^{2 *}$, LONGHUA LUO $^{1}$, KEZHAO ZHONG $^{1}$, \\ YUSHUI MA ${ }^{3}$, LINLIN FAN $^{4}$, DA FU $^{3}$ and LIJUAN WAN ${ }^{5}$ \\ ${ }^{1}$ Department of Urology, The First Affiliated Hospital of Nanchang University, Nanchang, Jiangxi 330006; \\ ${ }^{2}$ Key Laboratory of Stem Cell Biology, Institute of Health Sciences, Shanghai Institutes for Biological Sciences, \\ Shanghai Jiao Tong University School of Medicine/Chinese Academy of Sciences, Shanghai 200025; \\ ${ }^{3}$ Central Laboratory for Medical Research, Shanghai Tenth People's Hospital, Tongji University School of Medicine, \\ Shanghai 200072; ${ }^{4}$ Department of Pharmacy, Zhongshan Hospital, Fudan University, Shanghai 200032; \\ ${ }^{5}$ The Second Department of Internal Medicine, Cancer Hospital of Jiangxi Province, Nanchang, Jiangxi 330029, P.R. China
}

Received May 13, 2016; Accepted September 13, 2016

DOI: $10.3892 /$ or.2016.5159

\begin{abstract}
The identification of markers for disease diagnostic, prognostic, or predictive purposes will have a great effect in improving patient management. Proteomic-based approaches for biomarker discovery are promising strategies used in cancer research. In this study, we performed quantitative proteomic analysis on four patients including clear cell renal cell carcinoma (ccRCC) and paired adjacent non-cancerous renal tissues using label-free quantitative proteomics and liquid chromatography-tandem mass spectrometry (LC-MS/MS) to identify differentially expressed proteins. Among 3,061 identified non-redundant proteins, we found that 210 proteins were differentially expressed (83 overexpressed and 127 underexpressed) in ccRCC tissue when compared with normal kidney tissues. Two most significantly dysregulated proteins (PCK1 and SNRPF) were chosen to be confirmed by western blotting. Pathway analysis of 210 differentially expressed proteins showed that dysregulated proteins are related to many cancer-related biological
\end{abstract}

Correspondence to: Dr Da Fu, Central Laboratory for Medical Research, Shanghai Tenth People's Hospital, Tongji University School of Medicine, 301 Yanchangzhong Road, Shanghai 200072, P.R. China

E-mail: fu800da900@126.com

Dr Lijuan Wan, The Second Department of Internal Medicine, Cancer Hospital of Jiangxi Province, Nanchang, Jiangxi 330029, P.R. China

E-mail: 2563852741@qq.com

${ }^{*}$ Contributed equally

Key words: clear cell renal cell carcinoma, biomarker, prognosis, liquid chromatography-tandem mass spectrometry, proteomic profiling processes such as oxidative phosphorylation, glycolysis and amino acid synthetic pathways. Online survival analysis indicated the prognostic value of these dysregulated proteins. In conclusion, we identified some potential diagnostic biomarkers for ccRCC and an in-depth understanding of their involved biological pathways may help pave the way to discover new therapeutic strategies for ccRCC.

\section{Introduction}

Renal cell carcinoma ( $\mathrm{RCC}$ ) is the most common neoplasm in the adult kidney, accounting for 2-3\% of all malignant diseases in adults (1), and incidence rates are gradually increasing in most countries (2). Unfortunately, due to its asymptomatic development, $20-30 \%$ of the patients diagnosed incidentally with RCC during abdominal imaging already have advanced or metastatic disease and untreated patients with metastatic RCC have a 5-year survival rate of $<20 \%$ (3). The principal treatment for RCC is the surgical tumor mass removal, either partial or radical nephrectomy. However, surgery usually increases the duration of patient's life only for early stage tumors (4) and prognosis of patients with advanced stage or metastatic disease is poor (5).

WHO describes four main pathological subtypes of RCC: clear cell renal cell carcinoma (ccRCC), papillary carcinoma type 1 and 2, the chromophobic carcinoma and collecting duct carcinoma (3). The clear cell tumor subtype is the most common, accounting for $80-90 \%$ of all RCCs (2). The prognosis associated with ccRCC can vary widely and novel molecular prognostic markers are needed to assess prognosis at an earlier stage. A more in-depth understanding of the molecular basis and identifying new ccRCC biomarkers will be beneficial for cancer management.

Studying at protein level is desirable as mRNA levels do not always correlate well with the protein abundance. Proteomic-based approaches allow analyses not only at translational levels, but also at complex post-translational levels, which are not detected by gene analysis. Mass 
spectrometry (MS)-based proteomic approaches are well-suited for unveiling the complex molecular events of tumorigenesis and identification of cancer biomarkers. Among them, label-free methods make use of no isotope labels and therefore are simpler in sample preparation and lower in cost $(6,7)$. The past decade has witnessed a rapid increase in the use of label-free methods which show its potential for identification and quantification of differentially expressed proteins in normal and disease samples.

In this study, we aimed to identify potential tumor biomarkers through proteomic analysis. We performed quantitative analysis using label-free sample preparation and liquid chromatography-tandem mass spectrometry (LC-MS/MS) to identify proteins that are dysregulated in ccRCC. Then, we verified the dysregulated expression of several interesting proteins and assessed their clinical diagnostic significance of ccRCC.

\section{Materials and methods}

Ethics statement. The study was examined and approved by the Ethics Committee of the First Affiliated Hospital of Nanchang University. Each participant provided a written informed consent to participate in this study.

Patients and tissue samples. Samples of paired ccRCC and adjacent normal tissue were obtained surgically from four patients treated in the First Affiliated Hospital of Nanchang University after obtaining an informed consent. Relevant clinical information of the patients is summarized in Table I. None of the participants received chemo-, radio-, or immunotherapy before surgical resection. All specimens were histologically confirmed by two pathologists, and then in homogeneous areas were selected of the ccRCC samples to avoid grossly necrotic or fibrotic parts.

Sample separation by nano- $L C$ and analysis by tandem mass spectrometry (MS/MS). After nephrectomy, fresh ccRCC and adjacent normal tissues were cut on ice to homogenize in 20\% SDS and $1 \mathrm{M}$ DTT solution, following fluorescence assay (7) for total protein concentration. Approximately $100 \mu \mathrm{g}$ total protein from tissue was proteolysed on $10 \mathrm{kDa}$ filter (Pall Life Sciences, Shanghai, China) using a filter-aided sample preparation (FASP) protocol as described in detail elsewhere (8). Tryptic digests for each sample were quantitated by fluorescence assay. Peptide solution was then transferred to Empore Solid Phase Extraction Cartridge $(7 \mathrm{~mm} / 3 \mathrm{ml})$ for desalting and clean-up of sample. Peptide samples were resuspended in water with $0.1 \%$ formic acid (v/v) and analyzed by nano-LC-MS/MS.

For label-free, relative quantitative analysis, $5 \mu \mathrm{g}$ of the digest sample were analyzed by nano-LC-MS/MS, each sample was analyzed twice. LC separations were conducted on the EASY nano-LC system (Thermo Fisher Scientific GmbH, Bremen, Germany). Chromatography solvents were water (A) and acetonitrile (B), both with $0.1 \%$ formic acid. Peptide samples were concentrated and washed on a C18 ReversedPhase Trap Column $(75 \mu \mathrm{m} \times 2 \mathrm{~cm} ; 5 \mu \mathrm{m} ; 100 \AA$ A; Thermo Fisher Scientific $\mathrm{GmbH}$ ) with $0.1 \%$ formic acid, then they were eluted from the $\mathrm{C} 18$ analytic column $(75 \mu \mathrm{m} \times 15 \mathrm{~cm}$; $3 \mu \mathrm{m} ; 100 \AA$ A; Thermo Fisher Scientific $\mathrm{GmbH}$ ) with the following gradient $5-40 \% \mathrm{~B}(130 \mathrm{~min})$. At $140 \mathrm{~min}$, the gradient increased to $90 \% \mathrm{~B}$ and was held there for $10 \mathrm{~min}$. At $160 \mathrm{~min}$, the gradient returned to $5 \%$ to re-equilibrate the column for the next injection. Eluting peptides were directly analyzed via MS/MS on an LTQ Orbitrap Velos Pro mass spectrometer (Thermo Fisher Scientific $\mathrm{GmbH}$ ) equipped with a nano-electrospray ion source. A spray voltage of $1.8 \mathrm{kV}$ and an ion transfer tube temperature of $250^{\circ} \mathrm{C}$ were applied. The instrument was calibrated using standard compounds and operated in the data-dependent mode. The MS spectra were acquired in a data-dependent manner in the $\mathrm{m} / \mathrm{z}$ range of 350-1,800 and survey scans were acquired in Orbitrap mass analyzer at a mass resolution of 60,000 at $400 \mathrm{~m} / \mathrm{z}$. The MS/MS data were acquired in the linear ion trap by targeting the top 10 most abundant ions for fragmentation using low-energy collision-induced dissociation experiments, a normalized collision energy of $35 \%$, an activation q of 0.25 , and an activation time of $30 \mathrm{msec}$. MS scans were recorded in profile mode, while the MS/MS was recorded in centroid mode, to reduce data file size. Dynamic exclusion was set to a repeat count of one with a 30 -sec duration.

Data processing and analysis. All raw XCalibur files acquired from MS runs were analyzed using the default settings of MaxQuant software (version 1.3.0.5) with minor modifications. Enzyme specification during the search was trypsin/P. Carbamidomethylation of cysteine was selected as a fixed modification, while oxidation of methionine and $\mathrm{N}$-terminal acetylation were selected as variable modifications. Mass tolerances for precursor and fragment ions were set at $20 \mathrm{ppm}$ and $0.5 \mathrm{Da}$, respectively, in initial scan and set at $6 \mathrm{ppm}$ for the main search. Tandem MS search was done using the Andromeda search engine integrated into MaxQuant and was run against target databases against the Swiss-Prot human database (10/2015; 20,216 entries). Minimum cut-off for peptide length was set at seven amino acids, and maximum permissible missed cleavage was set at two. Maximal FDR for peptide spectral match, proteins and site was set to 0.01 . A minimum of two sequence-unique peptides was required for identification. Feature matching between runs was done with a retention time window of $2 \mathrm{~min}$ and the label-free quantification (LFQ) function was enabled. The MaxQuant peptide and protein quantification results from the 'peptides.txt' and 'proteinGroups. txt' files were imported into Perseus software (version 1.5.1.6) for further analysis. Statistical significance between the groups was assessed using one-way analysis of variance (ANOVA). Proteins were defined as differentially expressed if the ratios were $\geq 2$ or $\leq 0.5$ in RCC compared with adjacent normal tissue with a significant change $(\mathrm{p}<0.01)$.

Hierarchical clustering, Gene Ontology (GO) analysis, Kyoto Encyclopedia of Genes and Genomes (KEGG) pathway and protein interaction network analysis. Hierarchical clustering was performed using MEV software (v4.6, TIGR). The differentially expressed proteins $(\mathrm{p}<0.01)$ were analyzed by hierarchical clustering to find potential markers which can classify all samples.

Then, 210 dysregulated proteins were subjected to GO and KEGG pathway analyses by DAVID (http://david.ncifcrf. gov). Predicted protein-protein interaction networks for these 
Table I. Clinicopathological characteristics of four ccRCC patients.

\begin{tabular}{lcccc}
\hline Patient ID & Patient_W & Patient_H & Patient_L & Patient_P \\
\hline Gender & Female & Male & Female & Male \\
Age (years) & 11 & 57 & 73 & 62 \\
Histopathological type & ccRCC & ccRCC & ccRCC & Nephrectomy \\
Surgery & Nephrectomy & Nephrectomy & Nephrectomy & 4.5x4.5 \\
Tumor size (mm) & $6 \times 5$ & $7 \times 7$ & Single & Single \\
No. of foci & Single & Single & Good & Medium \\
Differentiation & Medium & Medium & T1N0M0 & T1N0M0 \\
TNM stage & T1N0M0 & T1N0M0 & Yes & No \\
Tumor infiltration & No & No & No & Yes \\
Smoking & No & & &
\end{tabular}

ccRCC, clear cell renal cell carcinoma.
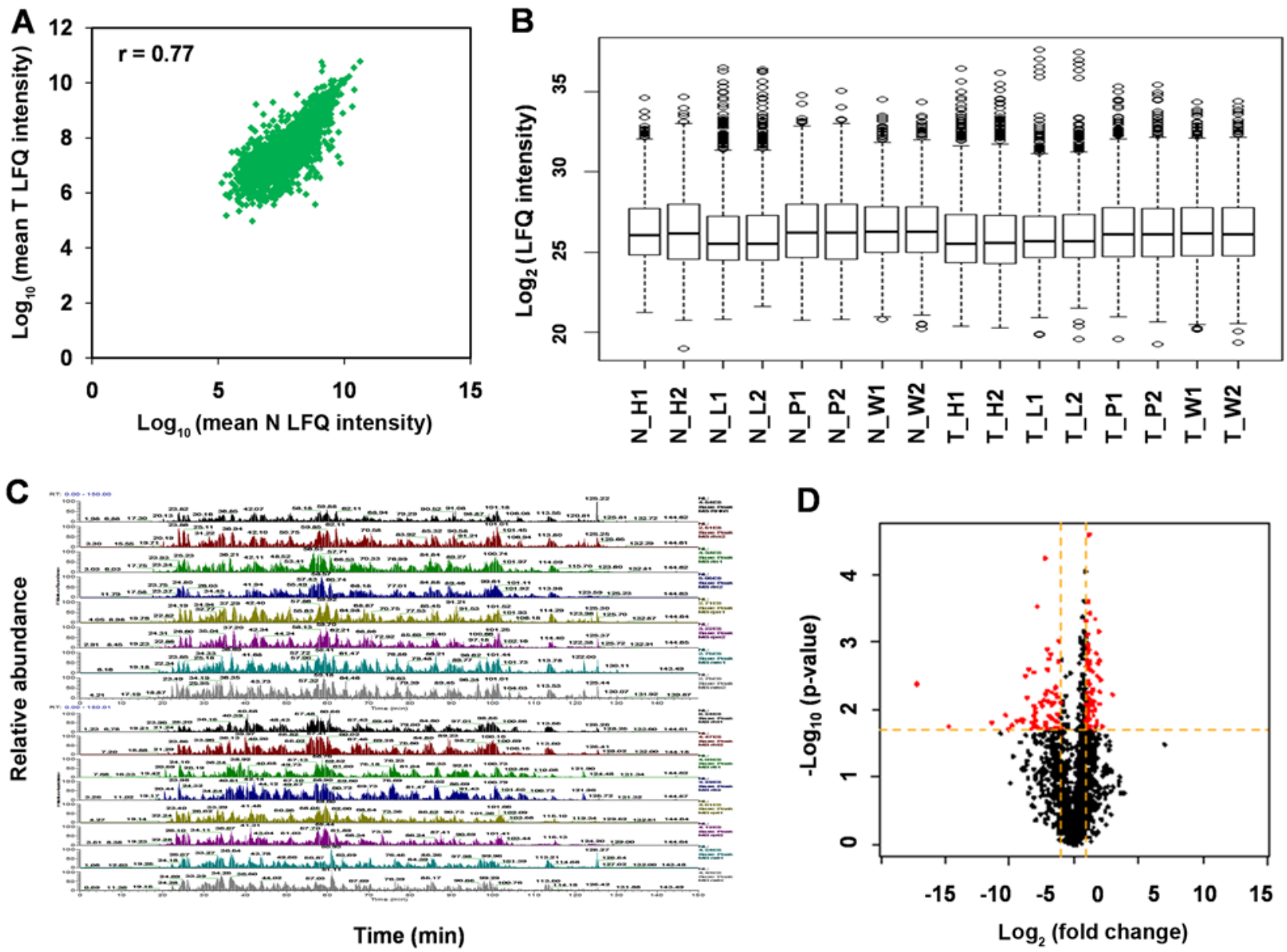

Figure 1. Analysis of sample nano-LC-MS/MS results and method evaluation. (A) Correlation of protein abundance (LFQ intensity) between normal RCC and normal kidney tissues. (B) Box plot of protein LFQ intensities from 16 nano-LC-MS/MS analyses. (C) Base peak mass spectrograms of 16 nano-LC-MS/MS analyses. (D) Volcano plot of protein expression differences between RCC tissue and normal kidney tissues as a function of statistical significance (standard $\mathrm{t}$-test, $\mathrm{p}<0.01)$ and $\log _{2} \mathrm{FC}>1$ or $<-1$ as indicated in red, proteins with no statistically significant difference in expression $(\mathrm{p}>0.01)$ and $\log _{2} \mathrm{FC}>-1$ and $<1$ are in black. LC-MS/MS, liquid chromatography-tandem mass spectrometry; LFQ, label-free quantification; RCC, renal cell carcinoma; FC, fold change.

210 differentially expressed proteins were performed by STRING (http://string-db.org/).

Western blot validation. Two most significantly dysregulated proteins (PCK1 and SNRPF) were chosen to be confirmed by western blotting. Briefly, $20 \mu \mathrm{g}$ of total protein were separated on a $10 \%$ SDS-PAGE gel. Proteins were then transferred to a PVDF membrane and probed with the following polyclonal antibodies: anti-PCK1 and anti-SNRPF (ab28455 and ab156587, 1:500; Abcam). GAPDH (sc-48166, 1:1,000; 


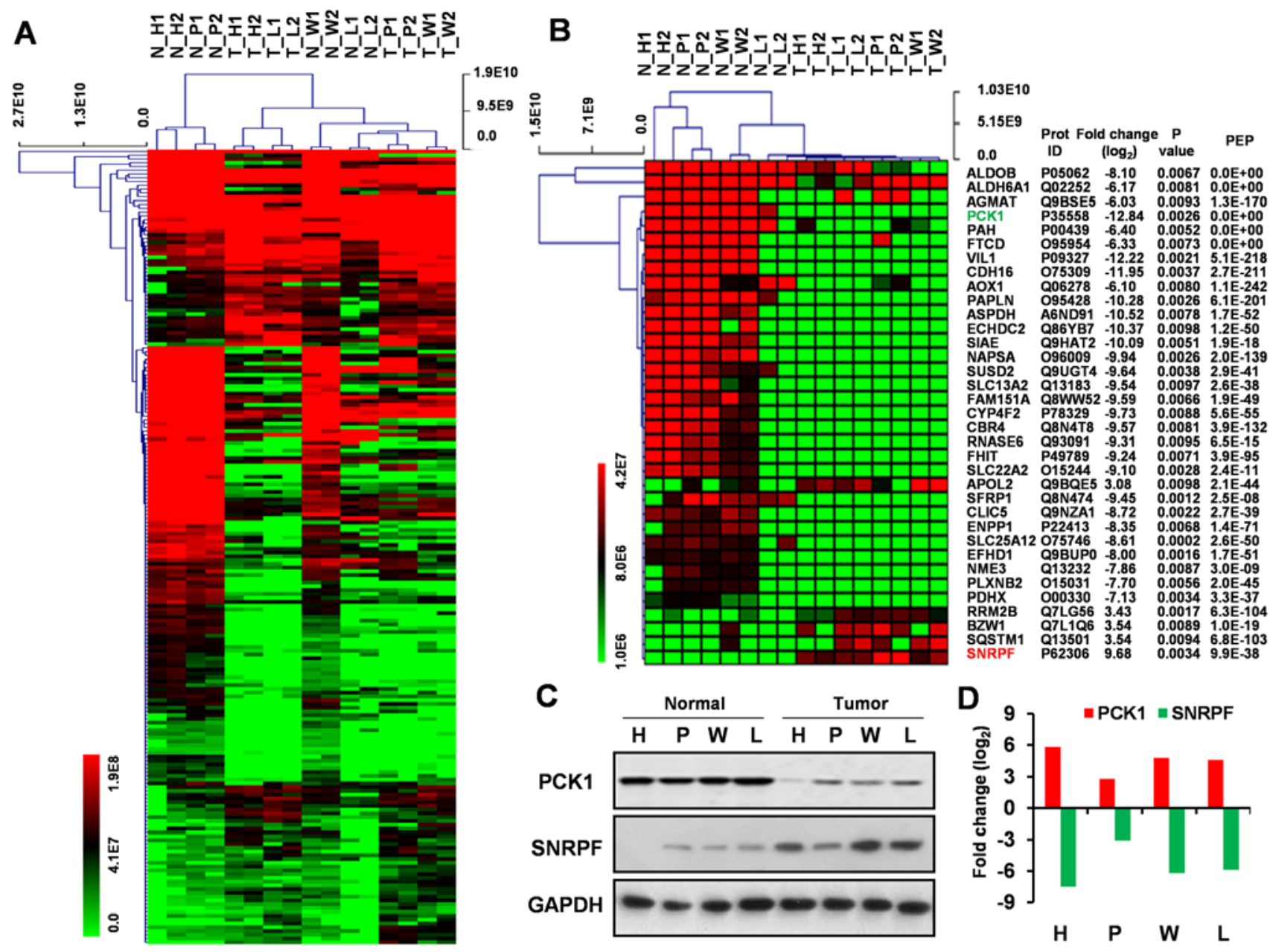

Figure 2. Hierarchical clustering analysis and validation of differentially expressed proteins. (A) Hierarchical clustering analysis of 210 dysregulated proteins in RCC compared with the normal renal kidney tissues. (B) Hierarchical clustering analysis of the top 35 up- and downregulated proteins in RCC compared with the normal renal kidney tissues. (C) Validation of PCK1 and SNRPF expression using western blotting. Representative blots showing the expression of PCK1 and SNRPF in four RCC tumor tissues and matched normal kidney tissues. GAPDH was used as a loading control. (D) Graphical representation of the $\log _{2} \mathrm{FC}$ in the expression of the two proteins between four RCC tumor tissues and matched normal kidney tissue as determined by densitometry. RCC, renal cell carcinoma; FC, fold change.

Santa Cruz Biotechnology, Inc.) was used as a loading control. Protein expression was visualized after incubation with secondary anti-rabbit antibodies conjugated with horseradish peroxidase and enhanced chemiluminescence reagent.

The intensity of protein staining was determined using Gel-Pro Analyzer 4.0. $\log _{2}$ fold change (FC) in expression of the two proteins between four ccRCC tumor tissues and matched normal kidney tissues is presented as a graph.

Set-up of differentially expressed genes for survival analysis. The expression levels of mRNAs were investigated in 47 paired ccRCC and normal tissue samples in the GEO dataset (GSE3-GPL10) from the NCBI platform (http://www.ncbi. nlm.nih.gov/). Five dysregulated genes (RPN1, CYP4F2, RPL27A, GSTM3, and DARS) showed the same trends with our proteomic data and were chosen to be investigated in renal cancer tissue samples in the TCGA database through the Oncomine database (http://www.oncomine.org). Univariate survival analysis of OS in RCC from the GEO database (GSE3-GPL10) as determined by Kaplan-Meier plot estimates based on five dysregulated expression genes.

\section{Results}

Identification of differentially expressed proteins in four paired ccRCC and tumor-adjacent kidney tissues. A total of four paired samples of ccRCC and tumor-adjacent kidney tissues was analyzed in the initial discovery phase. The same amounts of protein from each tissue were digested with trypsin, then the peptides were analyzed by nano-LC-MS/MS on a Orbitrap Velos Pro instrument. Scatter plot and box plot of protein abundance (LFQ intensity) between RCC tissue and normal kidney tissues show that protein expression variation between $\mathrm{T}$ and $\mathrm{N}$ is close ( $\mathrm{r}=0.77$ ) (Fig. 1A and $\mathrm{B}$ ). The base peak mass chromatograms show the good method repeatability of nano-LC-MS/MS (Fig. 1C).

Using MaxQuant, we identified a total of 3,061 non-redundant proteins with local FDR $<1 \%$ and at least two unique peptides per protein. To visualize the distribution of LFQ intensity ratios for the tissue proteins, a volcano plot of the $\log _{2}$ ratio of T/N vs. - $\log$ p-value was generated (Fig. 1D). The majority of proteins were showed to be at similar levels in the two tissues. 
Biological process

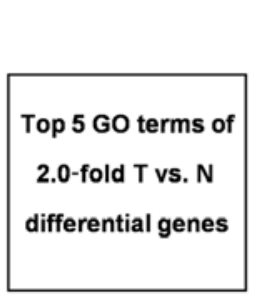

B
Molecular function

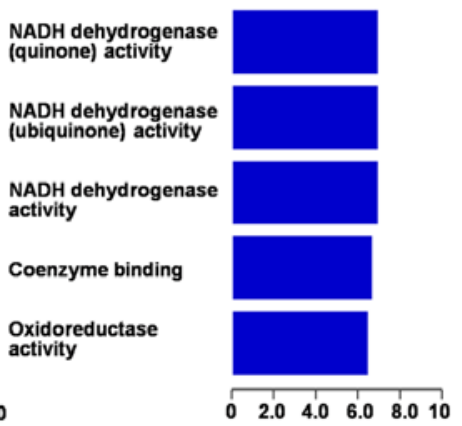

Enrichment score (- $\log _{10}$ (P-value))
Cellular component

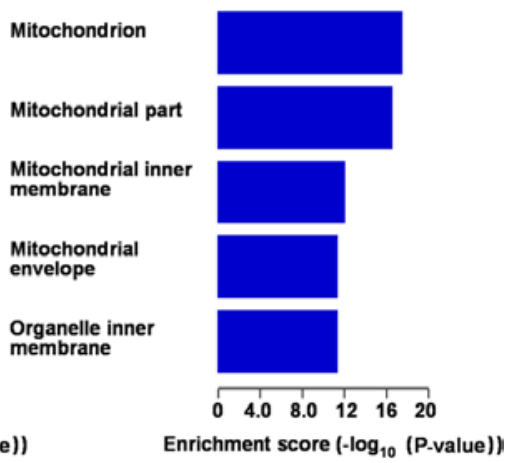

\begin{tabular}{|c|c|c|c|c|c|c|c|c|}
\hline Term & Count & $\%$ & $\begin{array}{l}\text { Pop } \\
\text { hits }\end{array}$ & $\begin{array}{l}\text { Fold } \\
\text { enrichment }\end{array}$ & P-value & Bonferroni & Benjamini & FDR \\
\hline hsa00190: Oxidative phosphorylation & & 0.47 & 130 & 5.05 & 3.91E-07 & 3.83E-05 & 3.83E-05 & 4.32E-04 \\
\hline hsa05012: Parkinson's disease & = & 0.44 & 128 & 4.81 & $1.89 E-06$ & $1.85 \mathrm{E}-04$ & 9.27E-05 & 2.09E-03 \\
\hline hsa03010: Ribosome & $\square$ & 0.35 & 87 & 5.66 & $6.26 \mathrm{E}-06$ & $6.13 E-04$ & $2.04 E-04$ & $6.92 E-03$ \\
\hline hsa05016: Huntington's disease & $\square$ & 0.47 & 180 & 3.65 & 2.36E-05 & 2.31E-03 & 5.77E-04 & 2.61E-02 \\
\hline hsa00330: Arginine and proline metabolism & 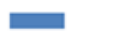 & 0.26 & 53 & 6.96 & 3.32E-05 & $3.25 E-03$ & $6.51 E-04$ & 3.67E-02 \\
\hline hsa00010: Glycolysis / Gluconeogenesis & $\square$ & 0.26 & 60 & 6.15 & 8.32E-05 & 8.12E-03 & 1.16E-03 & 9.20E-02 \\
\hline hsa00640: Propanoate metabolism & $\square$ & 0.21 & 32 & 8.97 & 9.59E-05 & $9.36 \mathrm{E}-03$ & 1.17E-03 & $1.06 \mathrm{E}-01$ \\
\hline hsa05010: Alzheimer's disease & ש & 0.38 & 163 & 3.27 & 5.15E-04 & 4.93E-02 & 5.60E-03 & $5.68 \mathrm{E}-01$ \\
\hline hsa00340: Histidine metabolism & $\square$ & 0.18 & 29 & 8.48 & 5.69E-04 & $5.42 \mathrm{E}-02$ & $5.56 \mathrm{E}-03$ & $6.27 \mathrm{E}-01$ \\
\hline hsa00020: Citrate cycle (TCA cycle) & ש & 0.18 & 31 & 7.94 & 7.83E-04 & 7.39E-02 & 6.96E-03 & 8.63E-01 \\
\hline hsa00380: Tryptophan metabolism & $\square$ & 0.18 & 40 & 6.15 & $2.55 E-03$ & 2.21E-01 & 2.06E-02 & $2.78 \mathrm{E}+00$ \\
\hline hsa00280: Valine, leucine and isoleucine degradation & ש & 0.18 & 44 & 5.59 & 3.90E-03 & 3.18E-01 & 2.90E-02 & $4.22 E+00$ \\
\hline hsa00650: Butanoate metabolism & - & 0.15 & 34 & 6.03 & 8.63E-03 & 5.72E-01 & $5.50 \mathrm{E}-02$ & $9.14 \mathrm{E}+00$ \\
\hline
\end{tabular}

Figure 3. GO and KEGG pathway analyses of differentially expressed proteins. (A) GO analysis of the top five GO terms of 2.0-fold T vs. N differential proteins associated with the biological process, molecular function and cellular component. (B) KEGG pathway analysis of 2-fold $\mathrm{T}$ vs. $\mathrm{N}$ differentially expressed proteins. GO, Gene Ontology; KEGG, Kyoto Encyclopedia of Genes and Genomes.

For the 3,061 proteins with LFQ intensity ratios calculated, significant expression differences in protein levels between the two tissues were determined by a Student's t-test $(\mathrm{p}<0.01)$ and by having a difference of $\geq 2$ or $\leq 0.5 \mathrm{FC}$ between tissues $\left(\log _{2}\right.$ LFQ intensity ratio $\geq 2$ or $\leq 0.5$ ). Among the 210 proteins exhibiting significant differences ( $>2$-fold difference, $\mathrm{p}<0.01$ ), there were 83 proteins with significantly decreased levels in ccRCC tissue vs. normal kidney tissues and 127 proteins with significantly increased levels in ccRCC tissue.

Hierarchical clustering for significantly dysregulated proteins. A unsupervised hierarchical cluster analysis was conducted on the significantly dysregulated proteins in ccRCC and the heatmap obtained from the analysis showed similar protein profile across four cases of RCC when compared with tumor-adjacent kidney tissues (Fig. 2A and B). Of all the proteins represented therein, 35 proteins displayed at least a 3 -fold increase or 6-fold decrease in expression (Fig. 2B).

Validation of dysregulated protein expression. We analyzed two most dysregulated proteins (PCK1 and SNRPF) using western blotting to validate our MS analysis using samples from the same RCC patient group. The expression of SNRPF was found to be upregulated and PCK1 downregulated in
ccRCC relative to normal kidney tissue when analyzed via western blotting, which coincide with MS data (Fig. 2C and D).

GO analysis. We next used GO to analyze 210 differentially expressed proteins (Fig. 3A). In biological process, the top five $\mathrm{GO}$ terms of 2.0 -fold $\mathrm{T}$ vs. $\mathrm{N}$ differential genes is oxidation reduction, generation of precursor metabolites and energy, electron transport chain, cellular respiration, and translation. In molecular function, the top five GO terms of 2.0 -fold T vs. N differential genes is NADH dehydrogenase (quinone) activity, NADH dehydrogenase (ubiquinone) activity, NADH dehydrogenase activity, coenzyme binding, oxidoreductase activity. In cellular component, the top five GO terms of 2.0 -fold T vs. N differential genes is mitochondrion, mitochondrial part, mitochondrial inner membrane, mitochondrial envelope, and organelle inner membrane.

KEGG pathway analysis. We used the online tool DAVID for finding enriched pathways for the 210 differentially expressed proteins. KEGG pathway analysis of these differentially expressed proteins between RCC and tumor-adjacent kidney tissues revealed some metabolism-related pathways including oxidative phosphorylation, glycolysis/gluconeogenesis and TCA cycle (Fig. 3B). 


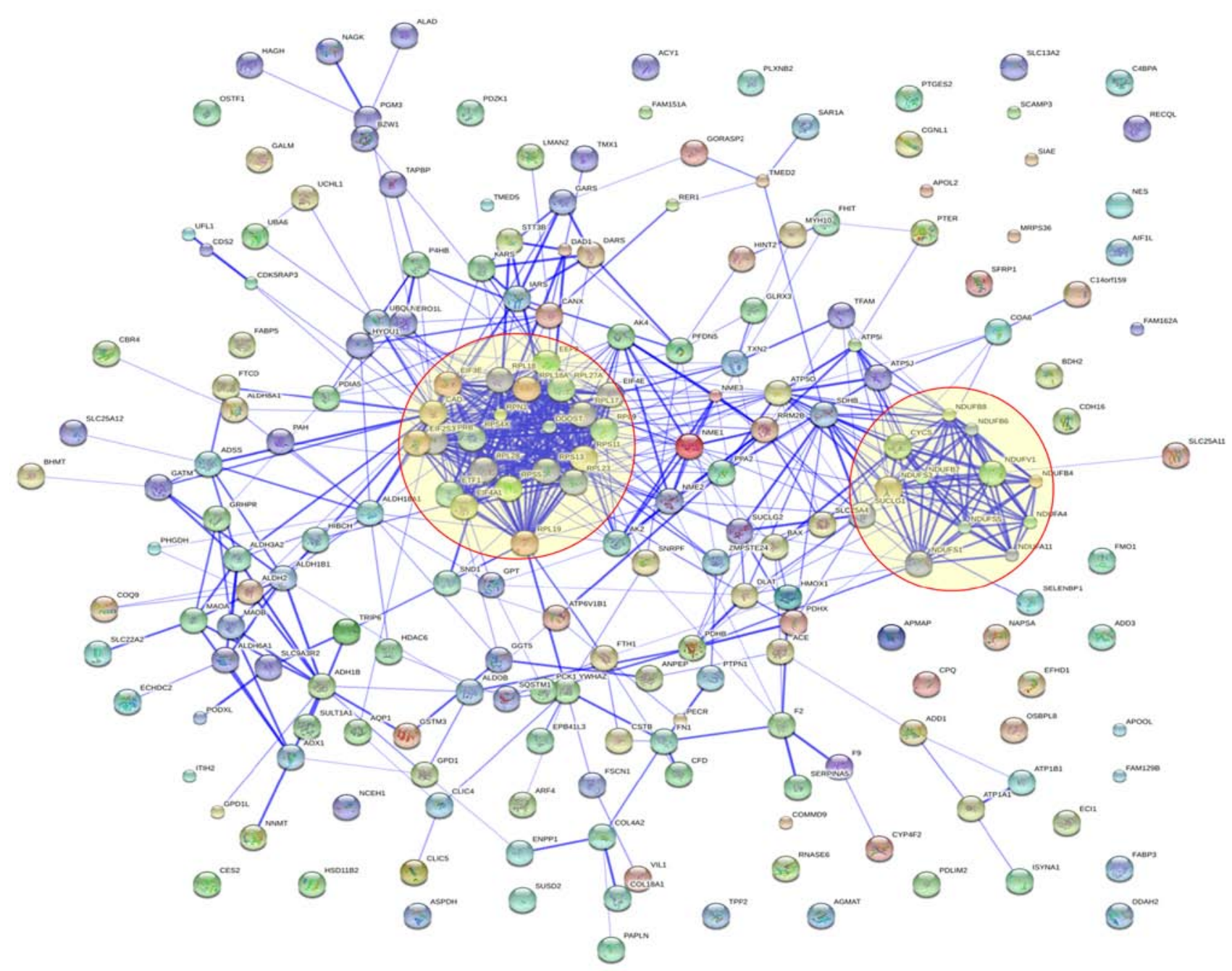

Figure 4. Visualization of protein-protein interactions for 210 dysregulated proteins using STRING analysis. Dysregulated proteins were used as input for STRING and are shown as spheres of different colors. Blue lines stand for interactions between proteins and the thickness of the lines represents the level of confidence associated with each interaction.

Protein interaction analysis. We also carried out protein-protein interaction analysis and found significant protein-protein interactions among these 210 dysregulated proteins. Several interaction groups were obvious and these interaction groups were labeled with orange circles. These proteins formed two main clusters: oxidative phosphorylation and ribosome protein (Fig. 4).

Identification of potential prognostic factors from dysregulated expression. To investigate the expression of these 210 dysregulated proteins and its potential prognostic significance, we downloaded a GEO dataset (GSE21362) from the NCBI platform (http://www.ncbi.nlm.nih.gov/), which included the mRNA microarry data from 47 paired normal kidney tissues and ccRCCs with follow-up data. We found a total of 24 dysregulated expression genes with $\mathrm{p}<0.05$ setting FC $>10$ (Fig. 5A). There are five significantly dysregulated genes, which are also found in our MS data. $R P N 1$ ( $\mathrm{FC}=162.42$, $\mathrm{p}=0.034), R P L 27 A(\mathrm{FC}=69.59, \mathrm{p}=0.034)$ and $D A R S(\mathrm{FC}=15.37$, $\mathrm{p}=0.038)$ gene in $\mathrm{RCC}$ tissue were significantly higher and CYP4F2 (FC=0.092, $\mathrm{p}=0.034)$ and GSTM3 $(\mathrm{FC}=0.004$, $\mathrm{p}=0.029)$ were significantly lower than in $\mathrm{RCC}$ relative to normal tissue ( $\mathrm{p}<0.001$, Fig. 5B).
We compared the mRNA expression of these dysregulated proteins using the Oncomine database. This analysis revealed that $R P N 1, R P L 27 A$ and $D A R S$ were upexpressed and $C Y P 4 F 2$ and GSTM3 were downregulated in tumor tissues when compared to normal tissues, which conform with our MS results.

To determine the prognostic value of these dysregulated expression genes in ccRCC, we used Kaplan-Meier survival analysis to analyze the dataset (GSE21362) to link gene expression with OS. The results showed that high expression of RPN1 ( $\mathrm{p}=0.029)$ and DARS $(\mathrm{p}=0.036)$ correlated with worsened OS, whereas high CYP4F2 $(\mathrm{p}=0.049)$ and GSTM3 $(p=0.048)$ levels were associated with increased OS (Fig. 5C), which indicate that our data from comparative proteomic profiling can identify some potential prognostic factors for human ccRCC.

\section{Discussion}

Early detection can significantly improve ccRCC patient outcome. The clinical diagnosis of asymptomatic ccRCC is often confirmed by imaging technology, such as CT and abdominal ultrasonography. However, there is currently no 

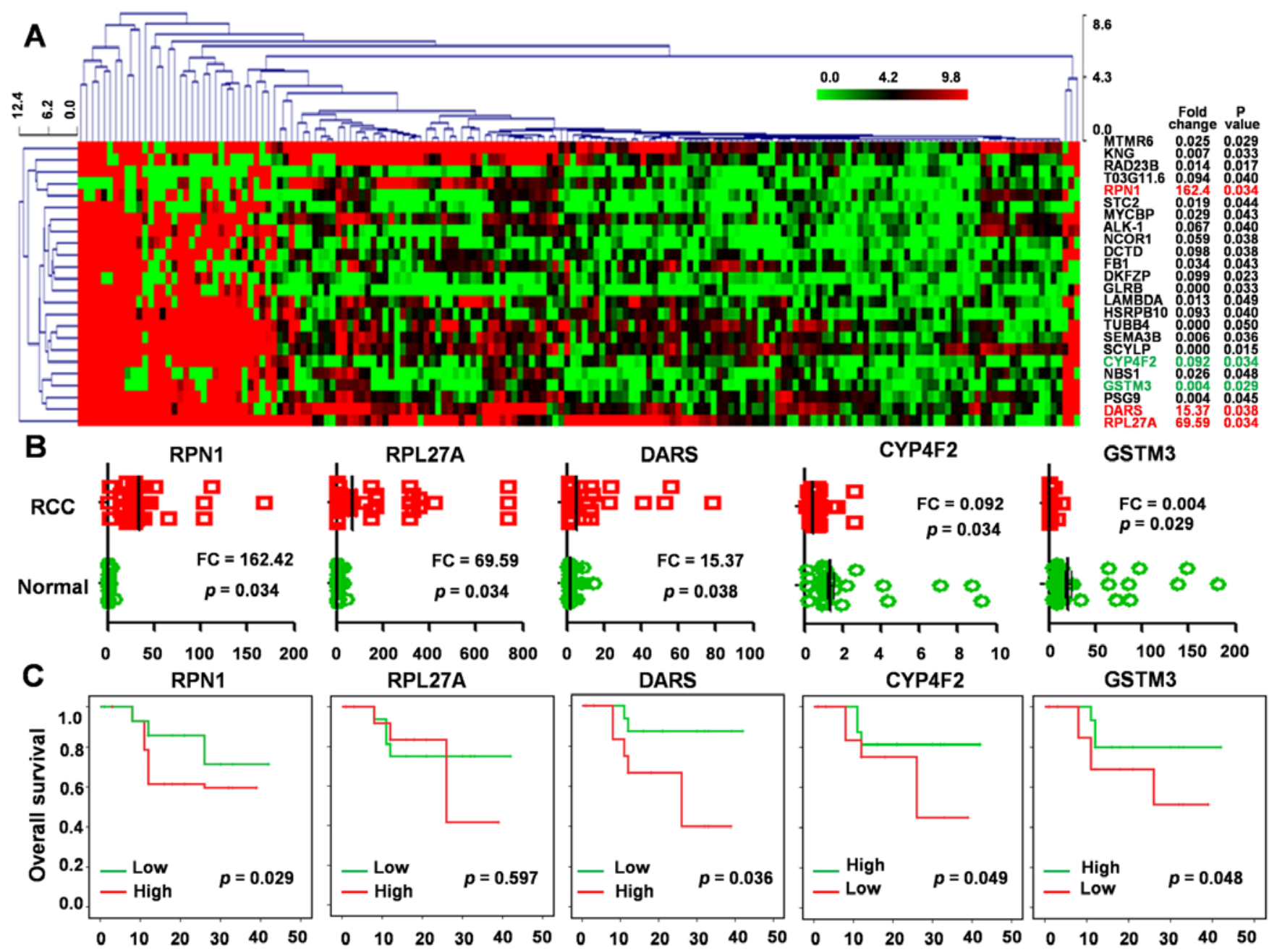

Figure 5. Validation of potential prognostic factors from dysregulated expression proteins. (A) Clustering analysis was performed using the MEV 4.7.1 based on 24 dysregulated expression genes (FC $>10, \mathrm{p}<0.05)$ in RCC and normal tissue ( $\mathrm{n}=47$ ) derived from the GEO database (GSE3-GPL10). The expression of five genes were consistent with our mass quantification analysis results. (B) The expression levels of five dysregulated expression genes in RCC vs. normal tissue derived from the GEO database (GSE3-GPL10) was analyzed. (C) Univariate survival analysis of OS in RCC from the GEO database (GSE3-GPL10) as determined by Kaplan-Meier plot estimates based on five dysregulated expression genes. FC, fold change; RCC, renal cell carcinoma.

validated biomarker to enable reliable screening for renal masses, whether benign or malignant (9). A more in-depth understanding of the molecular basis and identification of new RCC biomarkers would be beneficial for cancer management.

Most investigations to identify ccRCC-specific biomarkers were aimed to analyzing genes (10-12) or body fluid (e.g., urine, serum, and plasma) $(13,14)$. A considerable number of ccRCC-associated diagnostic or prognostic markers have been previously identified based on comparative analysis of ccRCC and normal kindey tissues, such as galectin-1, CNDP2, cabindin, gelsolin, heart fatty acid-binding protein and vimentin (9-14). However, these potential predictive or prognostic biomarkers require proper validation by appropriately designed randomized studies.

Proteomic-based approaches allow analyses not only at translational levels, but also at complex post-translational levels, particularly protein modifications like phosphorylation and glycosylation, which are not detected by gene analysis. MS-based proteomic approaches are well-suited for unveiling the complex molecular events of tumorigenesis and identification of cancer biomarkers.
There are several methods for protein separation and quantitative analysis of protein mixtures: two-dimensional polyacrylamide gel electrophoresis (2D-PAGE) followed by MS or MS/MS, stable isotope-labeling preparation coupled with LC-MS/MS, label-free preparation coupled with LC-MS/MS. However, for 2D-PAGE, it is difficult to detect proteins that are small $(<10 \mathrm{kDa})$, large $(>150 \mathrm{kDa})$, very basic (or acidic), hydrophobic, and remains a labor-intensive approach (15). Some limitations of the labeling approaches include increased sample preparation time, more complex methodology and higher costs attributed to labeling reagents, and only possible in several samples (16).

Label-free methods make use of no isotope labels and therefore are simpler in sample preparation and lowest in cost. It can also compare theoretically an unlimited number of treatment conditions (17). The past decade has witnessed a rapid increase in the use of label-free methods, which show its potential for identification and quantification of differentially expressed proteins in normal and diseased samples.

In this study, we aim to identify potential tumor biomarker through proteomic analysis in the tissue from renal patient 
cohort. We performed quantitative analysis using label-free sample preparation and LC-MS/MS to identify proteins that are dysregulated in ccRCCs compared to tumor-adjacent kidney tissues. The reliability and practicability of label-free proteomic analysis was confirmed by using western blotting to validate the two most dysregulated proteins. Hierarchical clustering analysis showed that these proteins can distinguish between normal and cancer tissue with accuracy. Furthermore, using GO and KEGG pathway analyses, we elucidated the potential involvement of these differentially expressed proteins in ccRCC pathogenesis. More importantly, our data provided some potential prognostic factors for human ccRCC.

\section{Acknowledgements}

This study was supported partly by grants from the National Natural Science Foundation of China (81372175, 81472501, 81472202 and 81302065). The funders had no role in the study design, data collection and analysis, decision to publish, or preparation of the manuscript.

\section{References}

1. Rini BI, Campbell SC and Escudier B: Renal cell carcinoma. Lancet 373: 1119-1132, 2009.

2. Znaor A, Lortet-Tieulent J, Laversanne M, Jemal A and Bray F: International variations and trends in renal cell carcinoma incidence and mortality. Eur Urol 67: 519-530, 2015.

3. Ljungberg B: The role of metastasectomy in renal cell carcinoma in the era of targeted therapy. Curr Urol Rep 14: 19-25, 2013.

4. Singer EA, Gupta GN, Marchalik D and Srinivasan R: Evolving therapeutic targets in renal cell carcinoma. Curr Opin Oncol 25: 273-280, 2013.

5. Nelson EC, Evans CP and Lara PN Jr: Renal cell carcinoma: Current status and emerging therapies. Cancer Treat Rev 33: 299-313, 2007.

6. Raimondo F, Corbetta S, Chinello C, Pitto M and Magni F: The urinary proteome and peptidome of renal cell carcinoma patients: A comparison of different techniques. Expert Rev Proteomics 11: 503-514, 2014
7. Boersema PJ, Aye TT, van Veen TA, Heck AJ and Mohammed S: Triplex protein quantification based on stable isotope labeling by peptide dimethylation applied to cell and tissue lysates. Proteomics 8: 4624-4632, 2008.

8. Neilson KA, Ali NA, Muralidharan S, Mirzaei M, Mariani M, Assadourian G, Lee A, van Sluyter SC and Haynes PA: Less label, more free: Approaches in label-free quantitative mass spectrometry. Proteomics 11: 535-553, 2011.

9. Pantuck AJ, Zisman A and Belldegrun AS: The changing natural history of renal cell carcinoma. J Urol 166: 1611-1623, 2001.

10. Feldstein MS, Rhodes DJ, Parker AS, Orford RR and Castle EP: The haphazard approach to the early detection of asymptomatic renal cancer: Results from a contemporary executive health programme. BJU Int 104: 53-56, 2009.

11. Siu KWM, DeSouza LV, Scorilas A, Romaschin AD, Honey RJ, Stewart R, Pace K, Youssef Y, Chow TF and Yousef GM: Differential protein expressions in renal cell carcinoma: New biomarker discovery by mass spectrometry. J Proteome Res 8: 3797-3807, 2009.

12. White NM, Masui O, Desouza LV, Krakovska O, Metias S, Romaschin AD, Honey RJ, Stewart R, Pace K, Lee J, et al: Quantitative proteomic analysis reveals potential diagnostic markers and pathways involved in pathogenesis of renal cell carcinoma. Oncotarget 5: 506-518, 2014.

13. Atrih A, Mudaliar MA, Zakikhani P, Lamont DJ, Huang JT, Bray SE, Barton G, Fleming S and Nabi G: Quantitative proteomics in resected renal cancer tissue for biomarker discovery and profiling. Br J Cancer 110: 1622-1633, 2014.

14. Raimondo F, Corbetta S, Savoia A, Chinello C, Cazzaniga M, Rocco F, Bosari S, Grasso M, Bovo G, Magni F, et al: Comparative membrane proteomics: A technical advancement in the search of renal cell carcinoma biomarkers. Mol Biosyst 11: 1708-1716, 2015.

15. Wang M, You J, Bemis KG, Tegeler TJ and Brown DP: Label-free mass spectrometry-based protein quantification technologies in proteomic analysis. Brief Funct Genomics Proteomics 7: 329-339, 2008.

16. Aebersold R and Mann M: Mass spectrometry-based proteomics. Nature 422: 198-207, 2003.

17. Old WM, Meyer-Arendt K, Aveline-Wolf L, Pierce KG, Mendoza A, Sevinsky JR, Resing KA and Ahn NG: Comparison of label-free methods for quantifying human proteins by shotgun proteomics. Mol Cell Proteomics 4: 1487-1502, 2005. 